its kinase activity. Inhibitors of $\mathrm{PI} 3 \mathrm{~K} \delta$ have been approved or are in clinical development for the treatment of B cell malignancies; their therapeutic potential in inflammatory and autoimmune disease is being explored. Seletalisib is a selective and potent PI3K $\delta$ inhibitor that has been profiled in preclinical and early clinical studies.

Objectives: To assess the therapeutic potential of seletalisib in inflammatory and autoimmune disease.

Methods: In vitro cell-based studies were performed on blood samples taken from healthy volunteers (HV) or from patients with primary Sjogren's Syndrome (pSS) and/or psoriasis. In addition, samples were analysed from a single ascending dose study in HV (NCT02207595) and a multiple ascending dose study in HV and psoriasis patients (NCT02303509). T and B cell responses in the presence and absence of seletalisib were assessed by flow cytometry or a Meso-Scale discovery assay (Meso-Scale Diagnostics, MD, USA), following activation of the T cell- or B cell-receptor by receptor cross-linking or via stimulation with specific antigens. The functional selectivity of seletalisib was assessed in the BioMap system (DiscoverX, CA, USA). Translational studies used flow cytometry and/or immunofluorescence to demonstrate the presence of active PI3K signalling in diseased tissue, through expression of phosphorylated-AKT (pAKT) or -ribosomal protein S6 (pS6). The effect of seletalisib on PI3K signalling was determined by phospho-flow cytometry. Target engagement was determined through measurement of basophil degranulation in healthy subjects, and assessment of lesion severity score in skin biopsies from patients with psoriasis that were treated with seletalisib.

Results: Preclinical studies showed seletalisib potently inhibited T cell differentiation and function ( $\mathrm{IC}_{50}$ range: 2-31 nM). Further, it blocked activation and proliferation of $B$ cells $\left(\mathrm{IC}_{50}\right.$ range: $\left.16-49 \mathrm{nM}\right)$. When profiled in a wide range of primary cell assay systems, including fibroblasts, epithelial, endothelial and vascular smooth muscle cells, seletalisib showed significant activity only in those systems containing lymphocytes, demonstrating its functional selectivity towards PI3K $\delta$-expressing cells. Expression of the PI3K pathway in lymphocytes was shown at the site of disease in clinical samples both from patients with psoriasis and pSS. Seletalisib inhibited PI3K signalling, measured by a reduction in PAKT and pS6 expression, in T cells derived from patients with psoriasis.

In first-in-man studies, mean seletalisib plasma concentration-time profiles increased with increasing dose after single and multiple dosing, with no major deviations from dose proportionality. There was no unexpected accumulation or loss of exposure after multiple dosing (time-independent pharmacokinetic (PK) profile) and apparent $t_{1 / 2}$ values (approx. 20h) were supportive of once-daily dosing. Inhibition of basophil degranulation in healthy subjects and effects on the cellular composition in lesional skin biopsies from patients with psoriasis, provided indications of target engagement, following treatment with seletalisib.

Conclusions: Seletalisib is a potent, selective PI3K $\delta$ inhibitor with an attractive preclinical and human PK profile; clinical studies are ongoing.

Disclosure of Interest: A. Payne Shareholder of: UCB Pharma, Employee of: UCB Pharma, M. Juarez Shareholder of: UCB Pharma, Employee of: UCB Pharma, G. Johnston Shareholder of: UCB Pharma, Employee of: UCB Pharma, E. Helmer Shareholder of: UCB Pharma, Employee of: UCB Pharma, M. Thomson Shareholder of: UCB Pharma, Employee of: UCB Pharma

DOI: 10.1136/annrheumdis-2017-eular.5132

\section{THU0221 DOWNREGULATED EXPRESSION OF MIR200B-5P IN MINOR SALIVARY GLANDS (MSG) OF PATIENTS WITH SJÖGREN'S SYNDROME (SS) ASSOCIATED LYMPHOMA}

E.K. Kapsogeorgou, A. Papageorgiou, M. Voulgarelis, A.G. Tzioufas. Pathophysiology, School of Medicine, National and Kapodistrian University of Athens, ATHENS, Greece

Background: The miRNAs of the miR-200 family are critical regulators of oncogene and tumor suppressor genes. Preliminary data from a limited number of patients with SS-associated lymphoma suggested that the expression of miR200b-5p in MSGs may be downregulated in lymphoma.

Objectives: To validate whether low miR200b-5p MSG-levels are associated with SS-related lymphomas and if they are deregulated before lymphoma development, suggesting a possible prognostic value.

Methods: miR200b-5p expression was analyzed by quantitative real-time PCR in total RNA from MSG tissues obtained from 77 SS patients and 9 patients with non-SS sialadenitis associated with sarcoidosis, HCV infection (4 each) or HBV (1 that was also diagnosed with MALT lymphoma). with chronic sialadenitis associated with sarcoidosis, HCV (4-each) or HBV infection (1 who was also diagnosed with MALT-Iymphoma). SS-patients included 28 that did not develop lymphoma during follow-up (without lymphoma; median follow-up time since biopsy performance, range: 6yrs, $1-12.75 y$ rs), 18 that developed lymphoma in the future (prelymphoma; median follow-up time till lymphoma diagnosis, range: $3.59 \mathrm{yrs}$, 0.42-8.5yrs, 15-MALT, 2-NMZL, 1-DLCBL) and 32 with SS-associated lymphoma at the time of biopsy (lymphoma; 25-MALT, 2-NMZL, 2-DLCBL, 1-BALT, 1-LP and 1 -SLL). In 15 cases, we had sequential MSG-samples from prelymphoma patients who transitioned to lymphoma (12-MALT, 2-NMZL, 1-DLCBL).

Results: Tukey's multiple comparison revealed that miR200b-5p levels were significantly down-regulated in MSG tissues of prelymphoma and lymphoma SSpatients (mean relative expression \pm SE: $0.37 \pm 0.10$ and $0.26 \pm 0.06$, respectively) compared to SS-patients without lymphoma $(0.67 \pm 0.07 ; p \leq 0.05$ and $p \leq 0.0001$ for pre- and lymphoma, respectively) or non-SS sialadenitis $(0.85 \pm 0.28, p \leq 0.05$ and $p<0.01$, respectively). Interestingly, low miR200b-5p levels were detected in HBV patient that had MALT Iymphoma (0.17). The expression of miR200b-5p was not found to differ between patients with SS without lymphoma and nonSS sialadenitis, or SS-associated pre-lymphoma and lymphoma. The analysis of the 15 cases of SS patients that had sequential samples before and on lymphoma diagnosis revealed that miR200b-5p levels do not significantly change over transition to lymphoma. The miR200b-5p expression levels were negatively correlated with the biopsy focus score $(r:-0.6550, p<0.0001)$, whereas they were not associated with the site or the number of involved sites, the type or the stage of lymphoma.

Conclusions: The significantly low levels of miR200b-5p in MSG tissues of patients with SS-associated prelymphomas and lymphomas suggest that miR200b-5p deregulation is implicated in SS-lymphomagenesis. The downregulation of miR200b-5p in prelymphoma samples and the lack of change over transition to lymphoma suggest that it can serve as a prognostic marker for future lymphoma development. The prognostic value of miR200b-5p in SS-associated lymphomas, the expressing cell types and affected molecular pathways are under investigation.

Acknowledgements: Funded by the Hellenic College of Rheumatology

Disclosure of Interest: None declared

DOI: 10.1136/annrheumdis-2017-eular.6708

\section{THU0222 PROPHYLACTIC AND THERAPEUTIC ADMINISTRATION OF AN ANTI-CD40 ANTAGONIST ANTIBODY BLOCKS AND REVERSES PROTEINURIA AND NEPHRITIS IN NZB/W-F1 MICE}

S. Perper, S. Westmoreland, Z. Liu, M. Duval, J. Seagal, B. McRae, S. Clarke. Abbvie, Worcester, United States

Background: The CD40-CD40L pathway is a potential target for the treatment of autoimmune diseases, as CD40 is a costimulatory receptor on antigen presenting cells (APCs) critical for the induction and maintenance of an immune response through binding CD40L on T cells. In the absence of CD40, both humoral and cellular responses to foreign antigens are severely impaired. In particular, disruption of this pathway prevents the development of disease in lupus-prone mice.

Objectives: The objective of this work is to evaluate whether prophylactic and therapeutic treatment with an antagonistic anti-CD40 antibody prevents and reverses nephritis and other manifestations of lupus in lupus-prone mice.

Methods: NZB/W- $F_{1}$ mice were i.p. administered anti-CD40 prophylactically or therapeutically at various doses once or twice per week. Prophylactic treatment of NZB/W- $F_{1}$ mice began at 26 weeks of age and continued for 9 weeks, while therapeutic treatment was initiated in NZB/W- $\mathrm{F}_{1}$ mice after they developed severe proteinuria (urine protein $>300 \mathrm{mg} / \mathrm{dL}$ ). Proteinuria was monitored weekly by urinalysis, and at study termination blood and spleen cells were analyzed by flow cytometry. Additionally, histological analysis of kidney, spleen, and salivary glands were performed, as well as gene transcription analysis of the kidney by microarray.

Results: As expected, prophylactic and therapeutic administration reduced the number of splenic germinal center (GC) B cells and T follicular helper cells (Tfh). Prophylactic treatment blocked development of proteinuria and extended survival in NZB/W- $F_{1}$ mice. Significantly, therapeutic anti-CD40 treatment reversed established, severe proteinuria in NZB/W- $\mathrm{F}_{1}$ mice and extended their survival. In agreement, the kidneys and salivary glands of treated mice exhibited reduced inflammation compared to control mice, and the kidneys exhibited lower CD40, IFN-I and chemokine gene signatures compared to controls. Interestingly, antiCD40 decreased expression of genes in kidney belonging to the "hepatic fibrosis" pathway, possibly explaining the improvement of kidney function.

Conclusions: Anti-CD40 treatment prevents the onset and reverses ongoing nephritis and sialadenitis in NZB/W- $\mathrm{F}_{1}$ mice. Resolution of ongoing nephritis results in restoration of low or normal urine protein levels. Thus, treatment with an antagonistic anti-CD40 is a strong candidate for clinical study in SLE.

Disclosure of Interest: S. Perper Employee of: Abbvie, S. Westmoreland Employee of: Abbvie, Z. Liu Employee of: Abbvie, M. Duval Employee of: Abbvie, J. Seagal Employee of: Abbvie, B. McRae Employee of: Abbvie, S. Clarke Employee of: Abbvie

DOI: 10.1136/annrheumdis-2017-eular.1583

\section{THU0223 ELEVATED MTORC1 SIGNATURE IN B CELLS FROM SJÖGREN'S SYNDROME PATIENTS CORRELATES WITH B CELL HYPERACTIVITY THAT IS ABROGATED BY MTOR INHIBITION: A NOVEL THERAPEUTIC STRATEGY TO HALT B CELL HYPERACTIVITY IN PSS?}

S.L. Blokland ${ }^{1,2}$, M.R. Hillen ${ }^{1,2}$, C.G. Kommer-Wichers ${ }^{1,2}$, A.A. Kruize ${ }^{1}$, J.C. Broen ${ }^{1,2}$, J.A. van Roon ${ }^{1,2}$, T.R. Radstake ${ }^{1,2} .{ }^{1}$ Rheumatology \& Clinical Immunology; ${ }^{2}$ Laboratory of Translational Immunology, UMC Utrecht, Utrecht, Netherlands

Background: A hallmark feature of primary Sjögren's syndrome (pSS) is B cell hyperactivity, including presence of autoantibodies, aberrant presence of $B$ cells and plasma cells in the salivary glands, elevated serum IgG levels and increased risk of lymphoma development. The mTOR pathway is essential for cell growth, 
survival and proliferation of B cells and mTOR inhibition has been shown to be effective in immune $B$ cell suppression in transplant patients and in treatment of $B$ cell lymphomas. Interestingly, in a pSS mouse model mTOR targeting inhibited lymphocytic infiltration in the lacrimal gland. However, mTOR activation in B cells has not been studied in pSS patients.

Objectives: To study the mTOR pathway in B cells of pSS patients as a potential therapeutic target to inhibit $\mathrm{B}$ cell hyperactivity.

Methods: Expression of mTOR pathway genes (MTOR, RPTOR, RICTOR, DEPTOR, AKT1, IGF1R, IGF1, PTEN) were assessed on an OpenArray platform in purified peripheral blood B cells and monocytes from pSS patients $(n=12)$, non-Sjögren's sicca patients $(n=17)$ and healthy controls ( $H C, n=9)$. Correlations with clinical parameters including lymphocytic focus score, ESSDAI and serum IgG levels were assessed. Flow cytometry analysis for B cell subset distribution was performed to assess potential effects of $B$ cell subset distribution on gene expression differences. Culture experiments were performed to study inhibition of the mTORC1 pathway (phosphorylated S6, kinase activity downstream of mTORC1) in association with inhibition of B cell proliferation and IgG production by mTOR inhibition.

Results: RPTOR and IGF1R expression were significantly increased in B cells from $p S S$ patients $(p=0.019$ and $p=0.018$, respectively) and correlated with serum IgG levels $(r=0.429, p=0.020$, and $r=0.462, p=0.012)$. Differences in expression of mTOR pathway genes were not found in monocytes. To indicate the mTOR signature a cumulative mTORC1 score was calculated consisting of $Z$ scores (AKT1 + IGF1R + IGF1 + RPTOR + MTOR - PTEN - DEPTOR) which was significantly elevated in $p S S(p=0.027)$, correlating with serum IgG levels $(r=0.463$, $p=0.011$ ). Frequencies of memory and naïve $B$ cells did not differ between $p S S$ patients and $\mathrm{HC}$ in this cohort $(\mathrm{p}=0.415)$. Activation of $\mathrm{B}$ cells in culture resulted in phosphorylation of $\mathrm{S} 6$, which indicates increased mTORC1 activity, in accordance with $\mathrm{B}$ cell proliferation and IgG production in both $\mathrm{HC}$ and pSS patients. Inhibition of mTOR by rapamycin decreased $\mathrm{pS} 6(\mathrm{n}=4, \mathrm{n}=2 \mathrm{HC}, \mathrm{n}=2 \mathrm{pSS}$, MFI $1400 \pm 335$ vs $935 \pm 306, p=0.060)$, strongly reduced $B$ cell proliferation $(n=6, n=3$ HC, $n=3$ pSS, $80.8 \pm 9.9$ vs $19.1 \pm 15.8 \%, p<0.001)$, reduced IgG+ $B$ cells $(n=6, n=3 H C$, $n=3$ pSS $39.5 \pm 15.5$ vs $11.1 \pm 5.7 \%, p=0.001)$ and decreased IgG production $(n=4$, $\mathrm{n}=2 \mathrm{HC}, \mathrm{n}=2 \mathrm{pSS}, 160 \pm 180 \mathrm{pg} / \mathrm{mL}$ vs $25.3 \pm 13.0 \mathrm{pg} / \mathrm{mL} \mathrm{p}=0.060$ ).

Conclusions: Presence of an mTORC1 signature in B cells from pSS patients correlating with B cell hyperactivity indicates a role for $\mathrm{mTORC} 1$ in $\mathrm{B}$ cell activation in this disease. The fact that $B$ cell proliferation and $\lg G$ production is effectively inhibited by rapamycin suggests that mTOR inhibition represents a potential therapeutic strategy for pSS.

Disclosure of Interest: None declared

DOI: 10.1136/annrheumdis-2017-eular.1616

\section{THU0224 ASSOCIATION OF IRAK-M WITH NEUROPSYCHIATRIC SYMPTOMS IN SYSTEMIC LUPUS ERYTHEMATOSUS PATIENTS}

S. Sacre, R. Thwaites, K. Davies. Brighton and Sussex Medical School, University of Sussex, Brighton, United Kingdom

Background: Systemic lupus erythematosus (SLE) is a chronic systemic autoimmune disease, where a breakdown in immune tolerance leads to sustained inflammation and tissue damage. Patients exhibit multi-organ involvement with a diverse range of symptoms that include arthritis, nephritis, neuropsychiatric events and dermatological complaints. Interleukin-1 receptor-associated kinase $M$ (IRAK-M), a negative regulator of toll-like receptor (TLR) signalling has previously been associated with SLE in a murine study [1]. Deficiency of IRAK-M was observed to exacerbated disease in a SLE model in C57BL/6-Ipr/lpr mice [2].

Objectives: This study aimed to investigate the expression of IRAK-M in monocytes from SLE patients compared with healthy control donors and measure downstream cytokine production upon TLR stimulation.

Methods: The study was approved by the Brighton East Research Ethics Committee and the National Research Ethics Service Committee North West - Lancaster. Whole venous blood was collected from 39 SLE patients and 19 healthy donors. Peripheral blood mononuclear cells were purified from whole blood after which monocytes were isolated using $\mathrm{CD} 14^{+}$selection beads. Expression of IRAK-M was determined relative to the geometric mean of the housekeeping genes glyceraldehyde 3-phosphate dehydrogenase and hypoxanthine phosphoribosyltransferase 1 by quantitative polymerase chain reaction. Cultured monocytes were stimulated with TLR ligands. Cytokine production was measured by enzyme-linked immunosorbent assay.

Results: IRAK-M expression was increased in the SLE patient group, however many of the patients did not show elevated expression compared to healthy donors. When stratified by disease symptoms, a clear correlation was observed between low expression of IRAK-M and neuropsychiatric symptoms which was not evident when evaluating other SLE symptoms. Interestingly, TLR activation led to elevated cytokine production in SLE patient monocytes that correlated with the basal level of IRAK-M expression in individual donors. Further investigation revealed that SLE monocytes demonstrated a reduced upregulation of IRAK-M after TLR activation compared to healthy donors.

Conclusions: Increased expression of IRAK-M was not unexpected in the SLE group due to the systemic inflammatory nature of the disease. However, not all patients presented with increased levels of IRAK-M and upon activation were less able to upregulate IRAK-M compared to healthy donors, suggesting a reduced capacity to resolve inflammation. This was reflected in the elevated cytokine production observed from SLE monocytes. Thus, low expression of IRAK-M in SLE monocytes is linked to elevated inflammatory cytokine production and may be a biomarker for neuropsychiatric symptoms in SLE patients.

References:

[1] Kobayashi, K., et al. Cell, 2002. 110(2): p. 191-202.

[2] Lech, M., et al. Ann Rheum Dis, 2011. 70(12): p. 2207-17.

Acknowledgements: Study funded by Brighton and Sussex Medical School.

Disclosure of Interest: None declared

DOI: 10.1136/annrheumdis-2017-eular.6201

\section{THU0225 ROLE OF THE IL-12/IL-35 BALANCE IN SJÖGREN'S SYNDROME}

O. Fogel $^{1}$, E. Rivière ${ }^{2}$, R. Seror ${ }^{2}$, G. Nocturne $^{2}$, S. Boudaoud ${ }^{3}$, B. Ly ${ }^{3}$ J.-E. Gottenberg ${ }^{4}$, V. Le Guern ${ }^{1}$, J.-J. Dubost ${ }^{5}$, J. Nititham ${ }^{6}$, K. Taylor ${ }^{6}$,

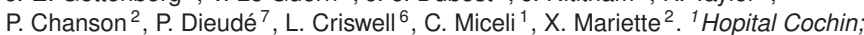
${ }^{2}$ Hopital Bicêtre; ${ }^{3}$ Inserm UMR1184, Paris; ${ }^{4}$ Hopital de Hautepierre, Strasbourg; ${ }^{5}$ Hopital Montpied, Clermont-Ferrand, France; ${ }^{6}$ UCSF, San Francisco, United States; ${ }^{7}$ Hopital Bichat, Paris, France

Background: An interferon (IFN) signature is involved in the pathogenesis of primary Sjögren's syndrome (pSS), but whether the signature is type 1 or 2 remains controversial. Mouse models and genetic studies suggested the involvement of Thelper 1 and type 2 IFN pathways. Likewise, polymorphisms of interleukin 12A gene (IL-12A), which encodes for IL-12p35, have been associated with pSS. IL-12p35 subunit is shared by 2 heterodimers, IL-12 and IL-35.

Objectives: To confirm the genetic association of $I L-12 A$ polymorphism and pSS and elucidate the involvement of the IL-12/IL-35 balance in pSS by functional studies.

Methods: The genetic study involved 673 patients with pSS from 2 French pSS cohorts and 585 healthy French controls. Functional studies were performed on sorted monocytes, stimulated or not. IL-12A mRNA and IL-12 and IL-35 protein levels were assessed by qRT-PCR and by ELISA and a multiplex kit for IL-35 and IL-12, respectively.

Results: We confirmed the association of the IL-12A rs485497 polymorphism and pSS and found an increased serum protein level of IL-12p70 in pSS patients carrying the risk allele $(p=0.016)$. Serum level of IL-12p70 was greater in patients than controls $(p=0.0001)$, especially patients with more active disease $(p=0.05)$; conversely IL-35 level was decreased in patients $(p=0.0001)$ especially in patients with a more active disease $(\mathrm{p}=0.05)$.

Conclusions: Our findings emphasize the involvement of the IL-12/IL-35 balance in the pathogenesis of pSS. Serum IL-35 level was associated with low disease activity, in contrast to serum IL-12p70 level, which was rather associated with a more active disease.

Acknowledgements: We thank Dr Odile Devergne (Université Paris Descartes, AP-HP, Hôpital Necker, Paris) for her expertise on the IL-35 ELISA kit to ensure the quality and reproducibility of this test.

The authors thank the following investigators of the ASSESS cohort prospective cohort of patients with Sjögren's syndrome (all in France) who recruited the patients and conducted follow-up: A. L. Fauchais (Limoges), S. Rist (Orleans), V. Le Guern (Paris), G. Hayem (Paris), J. Sibilia (Strasbourg), J. Morel (Montpellier), A. Saraux (Brest), A. Perdriger (Rennes), X. Puechal (Le Mans), E Hachulla (Lille) and V. Goeb (Rouen).

The authors thank Dr J. Benessiano, S Tubiana and all staff members of the Bichat Hospital Biological Resource Center (Paris) for their help in centralizing and managing biologic data collection from the French ASSESS,

Disclosure of Interest: None declared

DOI: 10.1136/annrheumdis-2017-eular.3586

\section{THU0226 DIFFERENTIAL SUSCEPTIBILITY OF TH17 AND T REGULATORY CELLS TO APOPTOSIS IN SYSTEMIC LUPUS ERYTHEMATOSUS PATIENTS - THE MODULATORY EFFECTS OF STATIN}

J. Sun, J. Frostegard, A. Liu. Institute of Environmental Medicine, Karolinska Institutet, Stockholm, Sweden

Background: Systemic lupus erythematosus (SLE) is a chronic autoimmune disorder. Patients with SLE have accelerated cardiovascular disease. Recent studies show there are more Th17 while less T regulatory (Treg) cells in the SLE patients. Th17/Treg imbalance may contribute to the pathogenesis of SLE.

Objectives: To investigate the underlying mechanisms of Th17/Treg imbalance, we test the proportion and susceptibility of Th17 and Treg to apoptosis, and the modulatory effects of statin in the SLE patients.

Methods: Totally 17 SLE patients and 20 gender- and age-matched control subjects were enrolled for this study. Peripheral blood mononuclear cells were isolated, either analyzed ex vivo, or cultured in the conditions to induce Th17 and/or Treg polarization. The proportion of Th17/Treg cells and frequency responding to apoptosis were analyzed by multiple color flow cytometry. Cytokines in cell culture supernatants and plasma were tested by ELISA. T cell polarization-related transcription factors were detected by quantitative real time PCR.

Results: The proportion of Th17 $\left(\mathrm{CD} 4^{+} \mathrm{IL} 17^{+}\right)$cells were higher in SLE patients, 